\begin{tabular}{|l|l|l||}
\hline \multicolumn{2}{|c|}{ PublisherInfo } \\
\hline \hline PublisherName & $:$ & BioMed Central \\
\hline \hline PublisherLocation & $:$ & London \\
\hline \hline PublisherImprintName & $:$ & BioMed Central \\
\hline \hline
\end{tabular}

\title{
SOCS1 mediates IL-6 effects
}

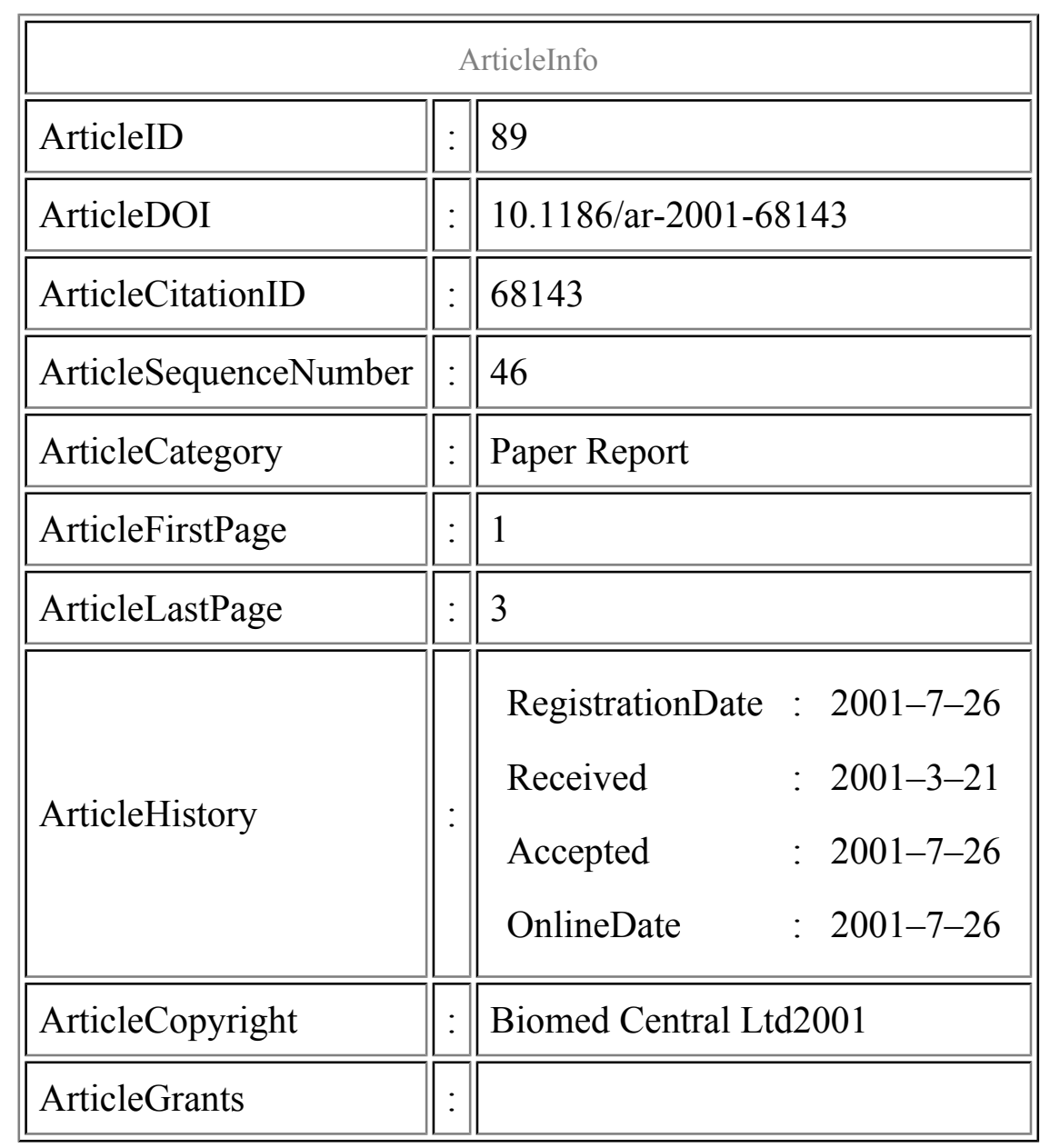




\begin{tabular}{|l|l|l|}
\hline ArticleContext & $:$ & 130753311 \\
\hline
\end{tabular}

Petya Dimitrova, ${ }^{\text {Aff1 }}$

Aff1 University of Erlangen, Germany

Keywords

IFN-?R, IL-6, SOCS1, Th1 differentiation

\section{Context}

Cytokines are the strongest regulators of T-cell differentiation. Interleukin (IL)-12 and interferon (IFN)-? drive Th1 differentiation whereas IL-4 promotes Th2 differentiation. It has been shown that IL-6 derived from antigen presenting cells initiates IL-4 production in naive $\mathrm{CD}^{+} \mathrm{T}$ cells which in turn polarizes them to the Th2 phenotype. In this study the mechanisms by which IL- 6 inhibits Th1 cell differentiation were evaluated.

\section{Significant findings}

A negative effect of IL- 6 on Th1 differentiation of $\mathrm{CD}^{+}{ }^{+} \mathrm{T}$ cells was demonstrated in different in vitro systems and in vivo. IL-6 reduced the level of IFN-? production in the presence or absence of exogenous IL-12. The presence of anti-IL-4 antibody did not prevent the suppressive effect of IL-6. Interestingly, IL- 6 did not influence IFN-? production by $\mathrm{CD}^{+} \mathrm{T}$ cells from mice deficient in IFN-? receptor a (IFN-?Ra). A decrease in IFN-?-induced STAT-1 phosphorylation in the presence of IL-6 was observed, suggesting that IL-6 might modulate Th1 differentiation by interfering with IFN-?R signaling. Consequently, the effect of IL-6 on IFN-? production by T cells deficient in suppressor of cytokine signalling 1 (SOCS1) and by wild-type cells was determined. While IL-6 inhibited IFN-? production by wild-type cells stimulated with anti-CD3 and anti-CD28, it failed to downregulate the production of

IFN-? in SOCS1-deficient CD4 ${ }^{+} \mathrm{T}$ cells. Moreover, IL-6 inhibited STAT-1 phosphorylation in $\mathrm{CD}^{+}{ }^{+} \mathrm{T}$ cells from IFN-? ${ }^{-/-} \mathrm{SOCS}^{+/+}$mice, but did not alter STAT-1 phosphorylation in T cells from IFN-? ${ }^{-/-}$ $\mathrm{SOCS1}^{-/-}$mice. 


\section{Comments}

IL-6 is produced by a variety of cell types such as macrophages, fibroblasts, endothelial cells, and activated T and B cells. IL-6 prevents resting T cells from undergoing apoptosis and regulates T-cell survival. Moreover, the systemic administration of IL-6 suppressed the development and the progression of experimental autoimmune encephalomyelitis, presumably by limiting the generation of Th1 effectors. The data in this study demonstrate that the negative effect of IL-6 on Th1 differentiation was independent of IL-4. IL-6 affected IFN-? production and IFN-?R signaling. Previous studies have demonstrated that IL-6-induced gene expression of the suppressor protein SOCS1 results in negative regulation of cytokine signaling. Thus, increased levels of SOCS1 might inhibit signaling triggered by IFN-?/IFN-?R ligation. In this regard, STAT-1 phosphorylation was prevented in CD4+ T cells from IFN-? ${ }^{-/-} \mathrm{SOCS}^{+/+}$transgenic mice. Together, the suppression of Th1 commitment by IL- 6 is mediated by SOCS1 via interference with IFN-?R signaling, and the effect of IL-6 on Th2 differentiation results from the initiation of IL-4 production. Therefore, the result of the pleiotropic functions of IL-6 is the modulation of Th1 or Th2 differentiation by two completely independent mechanisms.

\section{Methods}

ELISA, RNase protection assay, electrophoretic mobility shift assay

\section{Additional information}

\section{References}

1. Diehl S, Anguita J, Hoffmeyer A, Zapton T, Ihle JN, Rincon M: Inhibition of Th1 differentiation by IL-6 is mediated by SOCS1. Immunity. 2000, 13: 805-815.

This PDF file was created after publication. 